\title{
85598 - TUG SIMPLES E TUG DUPLA-TAREFA COGNITIVO-MOTORA COMO PREDITORES DO HISTÓRICO DE QUEDAS EM LONGEVOS: UM ESTUDO CASO CONTROLE
}

\section{Pôster - Gerontologia}

Fabiane de Oliveira Brauner / Brauner, F. O / PUCRS; Eliana da Silva Jaques / Jaques, E. S / PUCRS; Matheus de Souza Urbanetto / Urbanetto, M. S / PUCRS; Aniuska Schiavo / Schiavo, A / PUCRS; Marcos Silveira da Costa / Costa, M. S / PUCRS; Régis Gemerasca Mestriner / Mestriner, R. G. / PUCRS

Indrodução: Embora o teste do Timed Up and Go (TUG) seja amplamente utilizado em idosos, sua utilidade para a predição de quedas em longevos ainda não é bem estabelecida. Objetivo: avaliar a capacidade de predição dos testes Timed Up and Go (TUG) simples e em DT cognitivo-motora para o histórico de quedas em longevos não-institucionalizados. Metódos: estudo tipo caso-controle, a pesquisa foi aprovada pelo Comitê de Ética em Pesquisa da PUCRS (099196/2017). As avaliações ocorreram nas residências dos longevos, onde realizou-se seis tentativas válidas do teste TUG, sendo 03 em modo simples e 3 em DT, com o auxílio do sensor inercial G-Walk e um smartphone Motorola para filmagem dos testes. A amostra foi composta por 60 longevos com idade $\leq 85$ anos, (85-101) residentes na cidade de Porto Alegre. Dados sociodemográficos, níveis de atividade física, estado mental, presença de sintomas depressivos, preocupação com a ocorrência de quedas, fármacos em uso, autopercepção de equilíbrio funcional e o teste do alcance funcional também foram avaliados por instrumentos específicos. A caracterização da amostra deu-se por meio da estatística descritiva. A comparação de desempenho entre as modalidades do TUG S e DT) foi realizada pela ANOVA de medidas repetidas. Por fim, a predição da chance de quedas foi realizada com o emprego da regressão logística binária. Todas as análises foram realizadas no pacote estatístico SPSS 22.0. Resultados: os resultados demonstram que a maioria dos idosos incluídos na amostra eram fisicamente ativos e não possuíam alterações significatoivas no estado cognitivo conforme avaliação realizada pelo questionário do Mini Exame de Estado Mental. A realização do TUG em DT, embora reduza o desempenho dos idosos durante a realização do teste, não confere uma maior capacidade preditiva ao teste para diferenciar sujeitos caidores e não caidores. No entanto, a marcha não-linear composta pelo giro 1800 (fase de transição entre a marcha de ida e de volta) mostrou-se superior ao tempo total do TUG para a predição da chance de quedas, tanto em tarefa simples quanto em DT. Ser fisicamente ativo foi o principal fator protetor independente para a ocorrência de quedas. Conclusão: A adição de uma tarefa cognitiva ao TUG não melhora o valor preditivo do teste ao detectar a história de quedas em pessoas não institucionalizadas com 85 anos ou mais.

Palavras-chave: Acidente por quedas; Marcha; Longevidade, Controle Motor. 